\title{
LA LABOR DE LA TERAPIA OCUPACIONAL EN EL MARCO DE LOS DETERMINANTES SOCIALES DE LA SALUD EN CHILE
}

\author{
THE ROLE OF OCCUPATIONAL THERAPY CONCERNING THE SOCIAL \\ DETERMINANTS OF HEALTH IN CHILE
}

\section{Carmen Gloria Muñoz Muñoz'}

\begin{abstract}
Resumen
Las desigualdades en salud constituyen una prioridad creciente, en la medida que éstas se han hecho más evidentes y las estrategias implementadas, han fracasado. El resultado de la Comisión de Determinantes Sociales de la Salud impulsada por la OMS, estimuló la reflexión y entregó un avance en la interpretación conceptual; no obstante, este no es más que la ratificación institucional del tema, que en América Latina lleva décadas presente, con la explicación desde la medicina social y la salud colectiva. Críticas a las sugerencias hechas por la Comisión, giran sobre la falta de una aproximación concreta y territorial a las políticas sociales.

Así, el objetivo del artículo es pasar revista al documento de la OMS sobre Determinantes sociales de la salud, analizar las políticas sociales de Chile, para finalmente, someter a cuestión el rol de la Terapia ocupacional, que como disciplina del área de la salud, ha de contribuir en el desarrollo de estrategias que cumplan con lo esgrimido por el organismo internacional. El propósito es tensionar la reflexión sobre un eje insoslayable para el cumplimiento de lo planteado por la Comisión: la formación de una masa crítica sobre la cuestión social y política de los agentes de salud.
\end{abstract}

\section{Palabras clave:}

Terapia Ocupacional, determinantes sociales de la salud, política social, condiciones sociales, Chile (fuente: DeCS, BERIME).

\begin{abstract}
Health inequalities are a growing priority to the extent that these inequalities had become more evident and the strategies implemented had failed. The Social Determinants of Health Commision fostered by the WHO encouraged reflection and led to progress in conceptual interpretation. Nevertheless, this is only the institutional confirmation on the subject present for decades in Latin America through the explanation presented by social medicine and collective health. Some criticisms about the suggestions made by the Commision hinge around the lack of a concrete and territorial approach to public policy. The paper sets out to review the document on social determinants of health presented by the WHO and to analyze public policy in Chile, with the purpose of questioning the role of Occupational Therapy as a heath discipline that should contribute to the development of strategies that meet the international organization's proposals. The purpose is to add tension to the reflection about a key aspect to meet the points raised by the Commision: creating a critical mass of health agents oriented to social and political issues.
\end{abstract}

\section{Keyword:}

Occupational therapy, social determinants of health, public policy, social conditions, Chile (source: MeSH, NLM).

1 Terapeuta Ocupacional. Dra. en Salud Colectiva.

Docente Universidad Austral de Chile. Facultad de Medicina, Instituto de Aparato Locomotor y Rehabilitación.

Campus Isla Teja s/n, casa 2. Valdivia; Región de Los Ríos-Chile.

Fono: 56-063-2293510

cgmunoz@uach.cl 


\section{Los determinantes sociales de la salud}

La necesidad de disminuir las desigualdades en salud, parece una preocupación creciente para la Organización Mundial de la Salud (en adelante OMS), desde la emisión del lema-documento "Salud para todos en el Siglo XXI" en 1978 (Borrel \& Artazcoz, 2008; Baum, 2007) y desde el cambio, paulatino pero sostenido, del perfil epidemiológico mundial; ambos elementos presentan la urgencia de un mejoramiento sustancial de las condiciones de salud de los grupos más desfavorecidos. No obstante el tiempo transcurrido, y en virtud del incumplimiento de dichos propósitos, este se transformó en uno de los argumentos para que en el año 2005, iniciara trabajo la Comisión sobre Determinantes Sociales de la Salud (en adelante CDSS), que como organismo dependiente de la OMS, recabara datos científicos que a su vez, en calidad de evidencia, respaldaran pensamiento y acción sobre equidad en salud (OMS, 2008).

Presidida por Sir Michael Marmot del University College de Londres, trabajó ésta durante tres años y congregó a un total de 19 expertos del ámbito de la academia, investigación, formulación de políticas y lí- deres de la sociedad civil. América Latina contó con Ricardo Lagos Escobar, presidente de Chile entre los años 2000 y 2006 como único representante (OMS, 2008). Tras diez sesiones de trabajo y el respectivo proceso de consultas en las regiones con presencia OMS, el informe final fue dado a conocer en agosto del año 2008; contiene conclusiones y recomendaciones, además de un posicionamiento conceptual (Villar, 2007).

Como principal conclusión, se explicita en este documento, que la distribución desigual de poder, bienes, servicios e ingresos, combinados con el insuficiente resultado de la gestión política y programas sociales deficientes, afectan las condiciones de vida y específicamente, determinan las condiciones en que las personas nacen, crecen, viven, trabajan y envejecen, evidenciando notorias diferencias en la incidencia (frecuencia con que ocurre una enfermedad) y letalidad (probabilidad de morir de un enfermo) de la población que vive en determinados contextos sociales (OMS, 2008). La CDSS propone un modelo conceptual explicativo que combina una serie de elementos, que en interacción, evidencian las desigualdades y sus efectos (Borrel \& Artazcoz, 2008; OMS, 2008; Solar \& Irwin, 2010) (Figura 1).

Figura 1

Matriz explicativa de los Determinantes Sociales de la Salud, elaborado por Solar \& Irwin (2010) para Informe OMS.

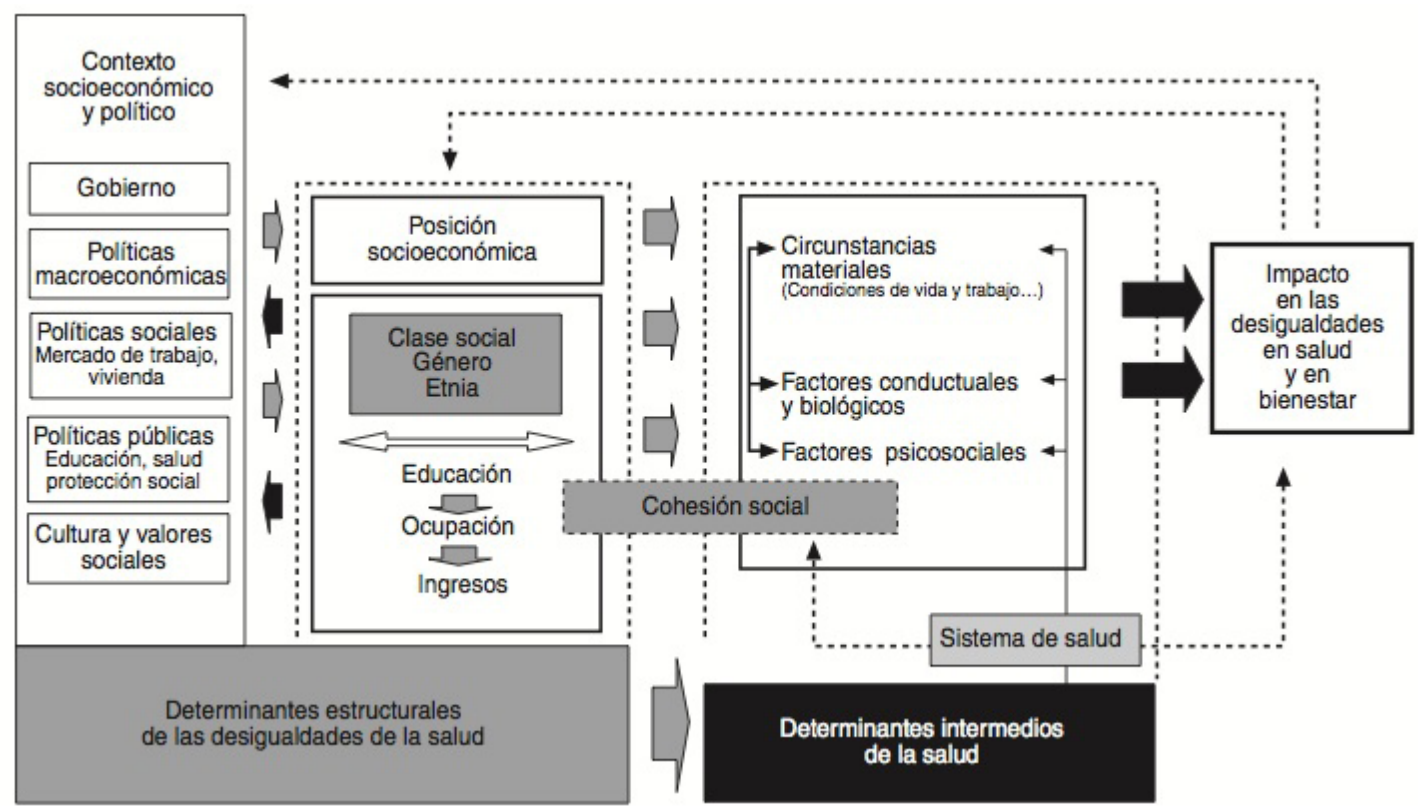

* Figura tomada de Borrell C., Artazcoz L. (2005, p. 466); original en inglés de Solar O., Irwin A. (2010, p. 6) 
La matriz presenta dos segmentos o dimensiones, en el primero de ellos se evidencian los "determinantes estructurales de las desigualdades de la salud" y en el segundo, los "determinantes intermedios de la salud"; los primeros incluyen el contexto socioeconómico y político particular (gobierno, políticas macro-económicas, políticas sociales, políticas públicas, cultura y valores sociales); mientras que los segundos, la posición socioeconómica donde, y en la medida que interaccionan etnia, género, clase social, determinan oportunidades diversas y/o desigualdades en educación, ocupación e ingresos, que no sería otra cosa que la expresión de jerarquías de poder en torno a recursos (Solar \& Irwin, 2010).

En la segunda dimensión, de los determinantes intermedios de la salud, se encuentran las circunstancias materiales (nivel de ingreso, acceso a recursos, condiciones de trabajo y/o vivienda entre otros), los factores conductuales/biológicos ("estilos de vida saludables") y los psicosociales (estrés, vulnerabilidad social, falta de redes). Los sistemas sanitarios aparecen con una determinación distinta a los tres puntos anteriores, no obstante se reconoce que el menor acceso, calidad y pago de servicio con "gasto de bolsillo" puede contribuir igualmente a las desigualdades en salud (Borrell \& Artazcoz, 2008; Villar, 2007).

La CDSS reconoce que las disparidades en las condiciones en que la agente nace, vive y muere, van más allá de la acción en el reducto sanitario. No obstante la equidad en el acceso a los servicios de salud es relevante, la solución pasa por el involucramiento del poder público, la sociedad civil, las comunidades locales y el sector empresarial (Borrell \& Artazcoz, 2008; Berlinguer, 2007). Para subsanarlas, recomienda tres líneas de trabajo: 1) mejorar las condiciones de vida; 2) luchar contra la distribución no equitativa del poder, el dinero y los recursos; 3) medir la magnitud del problema, analizarlo y evaluar los efectos de las intervenciones. Para cada una de ellas, a su vez, enuncia ámbitos de trabajo pertinentes y desarrolla en cada una de éstas una serie de estrategias (OMS, 2008) (Figura 2).

Figura 2

Propuesta de la CDSS para superar las desigualdades en salud.

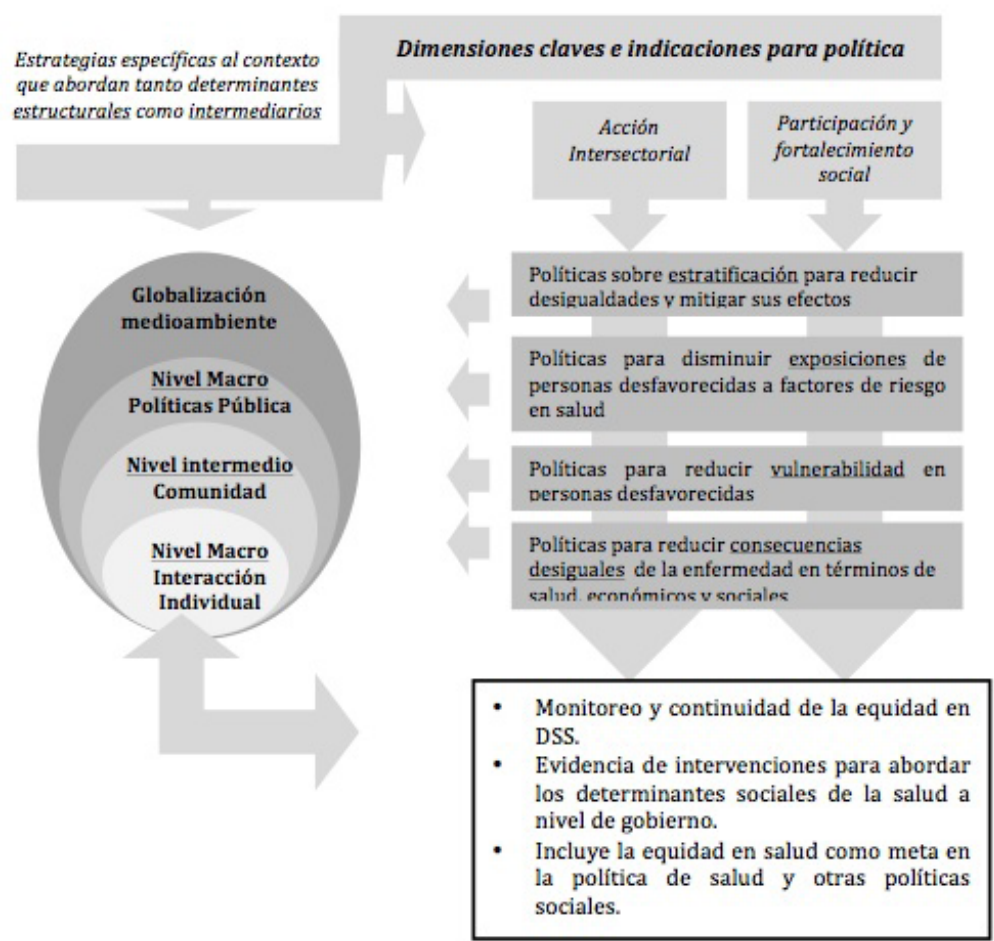

* Figura tomada de Solar O., Irwin A. (2010, p. 8). Traducción libre de la autora. 


\section{La crítica al informe de la CDSS de la OMS}

La CDSS reconoce que el origen de las disparidades en las condiciones en que la gente nace, vive y muere resulta de una política social insuficiente, mal aplicada, una estrategia económica injusta y una dirección política errada; la superación de las desigualdades sociales en salud (en adelante DSS) dejan de estar en el exclusivo reducto del sector sanitario, para involucrar a todos los ámbitos de poder públicos, la sociedad civil, las comunidades locales y el sector empresarial (Berlinguer, 2007). Se identifica el "empoderamiento para la salud" -entendido como las acciones promotoras que desafían la hegemonía establecida-, como poder para el cambio de las injusticias, para la organización y aumento de control sobre los acontecimientos que la determinan; en términos amplios, se critica el neoliberalismo y sugiere que el enfoque de las DSS debe ser construido e instalado desde la Atención primaria en salud (OSC, 2007; López, Escudero \& Carmona, 2008).

No obstante lo anterior, carece de una amplia reflexión sobre "la sociedad", que saque el tema de las desigualdades de un problema puramente redistribuido; fragmenta la realidad y pierde su dimensión sociohistórica, tras la reducción epidemiológica tradicional y dominante, que hace uso de las lógicas del riesgo y de la vulnerabilidad. Las recomendaciones parecen abstractas, silenciosas sobre condiciones generadas por la violencia, e invisibilizando los efectos del poder económico; la ética parece un imperativo para la eliminación de las desigualdades en salud, no obstante no existe una reflexión más abarcativa sobre la ética de la salud (OSC, 2007; López, Escudero \& Carmona, 2008).

No hay una reflexión profunda sobre la pobreza, ni las consecuencias de las desigualdades en la provisión de servicios de atención de la salud: la creciente debilidad de los sistemas públicos y el fortalecimiento de verdaderas cadenas de producción de salud privada (incluidas las farmacéuticas) (Villar, 2007; Berlinguer, 2007). Todos éstos, son aspectos largamente tratados desde perspectivas contra-hegemónicas como la salud colectiva y la medicina social latinoamericana, cuya interpretación requiere cruzar la línea de comprensión biológica individual o poblacional, para referirse a la determinación social a nivel colectivo, que ocurre con la generación de formas de apropiación, transformación, producción y posesión, que impactan y que tienen correlato con las condiciones de vida y salud (Berlinguer, 2007; OSC 2007; Behn, 2011; Alarzaqui, Mota \& Spinelli, 2007).
Sumado a lo anterior, la dispersión semántica sobre los conceptos de inequidad, iniquidad y desigualdad, arrastran consecuencias a las interpretaciones de las sugerencias y alcances de las mismas. Así por ejemplo, desde la perspectiva de la OMS -usando referencial y mayoritariamente los trabajos de Margaret Whiteheadconcibe linealmente inequidad, como desigualdades injustas, innecesarias y evitables, dejando las injustas como aquellas que no son resultado de la libre elección individual, sino que están fuera del rango de acción de los sujetos, como las biológicamente comprensibles por la fisiopatología. Desde esta perspectiva, todo lo socialmente explicable sería entonces, evitable-y por lo tanto-, injusto e innecesario. No obstante, el tratamiento en contextos reales de aquellos aspectos comprendidos como desigualdades, no es más que la manifestación concreta y medible de la inequidad; es decir, son empíricamente medibles, desde la evidencia de un trato desigual, comprensible desde las lógicas de las sociedades que la producen y cuya fuente primaria está en la apropiación diferencial de poder (Alarzaqui, Mota \& Spinelli, 2007; ALAMES, 2008).

\section{La recepción del informe sobre determinantes sociales de la salud en Chile}

En el discurso de los actores gubernamentales de salud en Chile (hacia el año 2008), tras el informe de la CDSS, se pueden identificar cuatro temas centrales, que relevan como logros cuatro aspectos: primero, que Chile es un país que avanza de manera sostenida en la provisión de mejores condiciones de vida de la población; segundo, que hay un reconocimiento de la importancia del factor social para las mejoras en las condiciones de salud y equidad, en donde la provisión de atención puede convertirse en sí misma en un generador de pobreza e inequidad, a través del gasto de bolsillo y otras barreras del acceso a la atención; tercero, que la solución de los problemas de la salud no tienen que ver ni única, ni predominantemente con ésta, y por lo tanto, requieren de articulación inter-sector; y finalmente, evidencian la necesidad de generar estrategias para superarlos, en donde la Reforma a la Salud (del 2005) es señalada como principal herramienta, en el marco más amplio de fortalecimiento del sistema de protección social (Barría, 2008; Vega, 2008).

En la base de este diagnóstico aparecen los resultados de los -hasta entonces incuestionables- instrumentos en uso en el país para medir la pobreza (principal- 
mente la encuesta CASEN). Estos evidenciaban el descenso progresivo de la pobreza, con el alza sostenible de empleabilidad y condiciones de vida en general. El análisis del sistema de protección social, como política gubernamental hacia 2008, mostraba estos logros como el resultado del balance entre las políticas públicas pro-crecimiento y redistributivas; entre el enfoque universal y de riesgo de las mismas; y las políticas sectoriales centradas en garantías a todos los ciudadanos, junto a programas específicos de apoyo a grupos vulnerables, en concordancia con las sugerencias de la CDSS y el enfoque de derechos (Barría, 2008).

En esta apuesta, el gobierno se propuso alcanzar igualdad de oportunidades para el logro de "progreso social" mediante una disminución de la inequidad en los salarios, acortando la brecha entre los mismos. Así, programas como Chile Crece Contigo y Chile Solidario, aparecen hoy como las estrategias que han permitido una mejora en el acceso y atención de las necesidades integrales de la infancia, donde se destaca por investigadores internacionales su valor, dada la intersectorialidad y articulación con otros programas gubernamentales que permiten (desde la planificación a la implementación), la participación activa de los sujetos atendidos, con una estrategia de intervención que articula comunidad, familia y beneficiario; y su modo articulado de la entrega de recursos, comparado con otras formas de asistencia social (Villar, 2007; FLACSO, OPS, MINSAL, OMS \& CDSS, 2005).

Apegados a los principios de la inter-sectorialidad para la construcción de políticas sociales propuestos por la CDSS, se crearon los Ilamados gabinetes sociales, que convocaron a los sectores de salud, educación, trabajo y protección social, planificación, y SERNAM, y sociedad civil, entre otros; más específicamente, la atención de salud se encauzó en la Reforma, que inició "marcha blanca" el año 2005, y cuyo principal soporte fue el entonces Ilamado Plan AUGE (aseguramiento universal de garantías explícitas en salud). Las garantías de este último son referidas -aún en la actualidad-al financiamiento, calidad y accesibilidad a toda la población -indistintamente usuario de sistema ISAPRE o FONASA-, cuyos diagnósticos estén incorporados al plan. Actualmente, el plan alcanza 86 patologías, y los reportes oficiales estiman que ha disminuido el "gasto de bolsillo" para el 77,9\% de la población (Barría, 2008).

Otras medidas en el marco de las sugerencias de la CDSS según fuentes gubernamentales cuentan: la rotulación obligatoria de alimentos, con información nutricional, previo a la venta; el desarrollo del plan de equidad de género 2006-2010 y las estrategias de participación ciudadana. Se propuso la revisión de programas de salud, orientados a fortalecer estrategias poblacionales que promuevan cambios de exposición y conductas de riesgo no saludables; así como el fortalecimiento de programas regionales de inclusión social hacia grupos de trabajadores con mayor desprotección y acciones integradas en zonas geográficas priorizadas como vulnerables (Vega, 2008).

En voz del Dr. Hernán Sandoval -uno de los ideadoresde la Reforma a la salud- ésta atendió a la necesidad de ponerle riendas a las injusticias sociales, propias de un "capitalismo puro y salvaje", transformando el tema en un imperativo ético y moral. Las "garantías" así, fueron concebidas como una forma de apropiación de poder de los beneficiarios, ante la erosión de derechos que los grupos de interés del complejo médico-industrial de la salud, pudiesen levantar al respecto (FLACSO y otros, 2005; Frenz, 2005). No obstante esto, una revisión detallada de los acontecimientos de la discusión política de la Reforma y su posterior implementación muestran de qué manera una de sus ideas fuerza -la de la solidaridad para el financiamiento- fue desmembrándose hasta quedar en un grupo de leyes que modificó el sistema administrativo y de atención que continúan estando lejos de la universalidad y equidad (Parada, 2005).

A juicio de algunos analistas, las políticas sociales en Chile -discursivamente ajustadas a las sugerencias de la CDSS- son posibles de clasificar en tres grupos: las sectoriales; las de desarrollo social y superación de la pobreza; y las de asistencia social focalizada (FLACSO y otros, 2005). Otras clasificaciones apuntan a organizarlas en aquellas tendientes a la disminuir las inequidades, mitigando los efectos de la pobreza, denominadas políticas de estratificación social; otras más bien intermedias o "preventivas", diseñadas para disminuir la exposición o vulneración de la población más desfavorecida; y un último grupo, más bien paliativo, destinado a reducir las consecuencias de las desigualdades o a fomentar su compensación (Vega, 2008; Frenz, 2005).

La más reciente reforma a la salud en Chile pone en evidencia -entre otras explicaciones por cierto- algunas de las críticas comentadas en el punto anterior a las sugerencias de la CDSS: se hace evidente la falta de una discusión que posicione constitucionalmente el lugar del derecho a la salud; la implementación del GES ha quedado reducida a un tema de control de gastos por sobre logros sanitarios, no como fuente de desarrollo que tienda a mayor equidad. La mal entendida inequidad en la 
provisión de servicios de salud no es más que evidencia de la desigualdad producida por un sistema dual: privado, desregulado, segregador y económicamente accesible para pocos; y uno público, insuficiente, endeudado y en desmantelamiento, para muchos.

Así, en un sistema de salud nacional que es en sí mismo inequitativo, es comprensible para algunos que GES, y programas como Chile Solidario o Chile Crece Contigo constituyan avances; pero por ejemplo, si bien GES -como política de salud-, ha pretendido nivelar las posibilidades de tratamiento (indistintamente usuarios de sistema público o privado), ha generado nuevas desigualdades: las listas de espera GES no se encuentran precisamente en el sistema privado, sino en el público. La más evidente y dramática está dada ahora entre quienes son diagnosticados con un cuadro GES y uno no GES.

La realidad actual de la política social en Chile evidencia la ausencia de aquellas que propongan la erradicación de la inequidad que genera desigualdades; una forma de organización social, política y económica que tienda a una sociedad que no genere nuevas inequidades; $i$ es posible tener un sistema de salud equitativo en una sociedad que no juzga y naturaliza la inequidad social? Un camino conducente a la superación de los DSS, debiera posibilitar la intersectorialidad y participación vinculante de distintos actores sociales; un Estado fuerte en la regulación de la producción, distribución y financiamiento; y una sociedad civil que de manera activa medie la tensión entre el mercado y el Estado.

En otro terreno, si bien es sabido que las tasas de mortalidad en la región han disminuido y las enfermedades infecto-contagiosas han sido controladas del cuadro etiológico de gran parte de la región, estas no han hecho otra cosa que ceder terreno frente a la obesidad, la enfermedad crónica-degenerativa, a los problemas de salud mental, el abuso de sustancias, y a las muertes por accidentes. Si bien este corresponde a lo que algunos han denominado un fenómeno propio de la modernidad, es irreal creer que somos un país más sano; enfermamos de otras cosas y eso dista mucho de lo primero, siendo el perfil epidemiológico actual chileno perfectamente explicable desde la inequidad y la desigualdad en salud entre otros aspectos).

\section{La terapia ocupacional en el marco de la cuestión de los determinantes sociales de la salud}

Actualmente, parecemos consensuar con respecto a que la TO es una disciplina aún en desarrollo, con una estructura epistémica laxa, que se ha construido desde la praxis, con un claro predominio teórico extralatinoamericano, en donde el trabajo en ciencias de la ocupación ha permitido, lentamente, la reflexión constructiva sobre este mismo hecho y eso, ha constituido la oportunidad de desarrollos conceptuales propios (Guajardo, 2010). En ese sentido, la reflexión sobre el tránsito, desde el paradigma biomédico al de la complejidad, hacen posible entender de mejor manera que las necesidades de los sujetos van más allá del tránsito entre la salud-enfermedad-que nos permite el paradigma mecanicista-, para preocuparnos entonces de las potencialidades, habilidades y oportunidades para la vida y el bienestar de los mismos (Morrison, Olivares, Vidal, 2011).

El "colonialismo teórico anglosajón" (Guajardo, 2010), se ha visto permeado a las reflexiones y las prácticas de la disciplina en forma significativa, contextualizando de mejor manera éstas, con un sentido históricoterritorial; así, discutimos sobre pertinencia cultural de las intervenciones; de la naturaleza ocupacional de los sujetos y del rol político-social de la disciplina, entre otros aspectos. Es más, recientemente se trabaja sobre la idea de la emergencia de un nuevo paradigma de la ocupación: el social. Este cambio epistemológico, supondría dejar de ver el fenómeno de la ocupación como algo individual, para comprenderlo en una dimensión sistémica, compleja, que incluye lo económico, lo político, sanitario, cultural, social, en coherencia con la justicia y el bienestar de comunidades (Morrison, Olivares, Vidal, 2011).

Asistimos, entonces, al enorme desafío de poner rostro, pensamiento crítico y por sobre todo, propuestas a una praxis que evidencie la ocupación como un fenómeno arraigado en las condiciones de vida, territorial e históricamente contextualizadas y que también históricamente, rehuimos nombrar (o nombramos con dificultad) como ejes de nuestra práctica. La praxis disciplinar (reflexión más acción) sobre "lo social": de y en lo social, no nos puede distanciar del fenómeno de la ocupación, como eje central de la disciplina, sino que enriquecer la discusión sobre ello con una tensión profunda sobre las implicancias y significados del mismo/en ella misma. Esto requiere: debatir e intercambiar sobre los problemas y desafíos contemporáneos mundiales, latinoamericanos y nacionales; pero entender que los saberes abstractos no quedan en nada, si no lo posicionamos sobre realidades políticas, económicas e históricas concretas; que no son otra cosa que aquello que media las necesidades de interventores y usuarios. 
Entonces, si la ocupación es el fin o el medio a través del cual los seres humanos mantenemos salud, bienestar, función (apostando al amplio espectro de paradigmas), debiese resultarnos un imperativo ético pensar sobre la realidad actual de la provisión de servicios de salud en el país; los obstáculos y posibilidades que ha brindado la protocolización de intervenciones para la esencia y principios fundantes de la disciplina y la práctica centrada en el cliente (entre otros aspectos); la forma de priorización para la atención que se establece para los usuarios (patologías GES y no GES); las lógicas de funcionamiento del sistema privado en salud, principalmente referido a altos costos y la discriminación que imponen los mismos; el acceso universal de prestaciones de la disciplina (y nuestra reiterada necesidad de entrar en modalidad libre elección de FONASA).

En otro terreno de cosas, hacer una terapia ocupacional consecuente con las sugerencias de la CDSS, requiere pensar, investigar, tomar postura e intervenir en base a lo que significa la precarización del trabajo; las condiciones económico-sociales de los adultos mayores una vez jubilados bajo el actual sistema de pensiones en régimen en Chile; las precarias posibilidades de inclusión educacional, laboral y social de personas con discapacidad; la realidad actual de las personas privadas de libertad en contextos de cárceles concesionadas por el Estado; la violencia, represión y discriminación que afecta en lo cotidiano a pueblos originarios y migrantes; el acceso y calidad de la educación como principal herramienta de equidad social, entre otros.

Todos estos enunciados cuentan con un correlato en el mundo de la ciencia de la ocupación, e impactan el trato cotidiano en el quehacer práctico; constituyen aquello denominado discriminación, deprivación, desequilibrio, justicia/injusticia ocupacional; inclusión y exclusión social u ocupacional (Crepeau, Cohn \& Schell, 2009; Kronenberg, Simó \& Pollard, 2005). Los DSS son una tarea pendiente en el concierto internacional, para la World Federation of Occupational Therapists, que si bien define posiciones con respecto a los derechos humanos, la práctica centrada en el cliente y la Rehabilitación con base comunitaria (WFOT, 2011), entre otros aspectos, no lo hace aún sobre la inequidad social y la desigualdades en salud, tema trascendente si consideramos que la labor del federalismo es posicionar la importancia de la práctica disciplinar y su contribución a la sociedad.

No comprenderemos la ocupación de los sujetos con quienes trabajamos si no entendemos qué estructu- ra los soporta, ni qué estructura los desestructura; con esto digo, que la ocupación no está en el aire ni sólo en el contexto social, está en una arena donde, en una lucha de poder, se definen las formas de nacer, enfermar, sanarse, morir y sobrevivir; ser feliz o desdichado. La ocupación constituye parte de esa dinámica, forma parte de lo cotidiano, pero también de lo estructural. Nuestro entorno "social" construye -si no queremos ser radicales, para decir que "determina" el involucramiento en ocupaciones, por lo tanto, si ese ha de ser nuestro objeto de estudio, entender "lo social" pasa por entender las oportunidades, necesidades y desafíos que este impone a la gente; subsanar los determinantes sociales de salud es una tarea entonces de la que no podemos quedar al margen, y debemos llevarlo a nuestra competencia tanto en la realidad de nuestros usuarios como a las del gremio mismo.

Requerimos por tanto, de profesionales terapeutas ocupacionales capaces de pensar sobre la contingencia social, de entender cómo la globalización, la sustentabilidad del planeta, la pobreza y la marginación; las forma de producción, las crisis económicas, las fuentes y condiciones de (des)empleo, los desastres naturales, las violaciones a los derechos humanos, y de las libertades individuales, afectan una forma colectiva e individual de enfrentarnos a la vida. Desde mi particular punto de vista, una sociedad que gesta inequidad nunca será capaz de superarla, por mucha y buena política social que cree; la solución está en generar sujetos sociales con potencial y gusto por la transformación social, que en sus procesos de trabajo y mediante apropiación de poder -democráticamente responsable-, permeen los macro sectores sociales desde aquello que acontece en un box de atención, en una sala de clases, de rehabilitación, o en un taller vecinal.

\section{REFERENCIAS BIBLIOGRÁFICAS}

Alarzaqui, M.; Mota, E.; Spinelli, H. (2007). El abordaje epidemiológico de las desigualdades en salud a nivel local. Cad Saúde Pública, 23(2), 321-330. Extraído el 4 de mayo 2014, desde: http://www.scielo.br/pdf/csp/v23n2/08.pdf

Asociación Latinoamericana de Medicina Social (2008). Taller Latinoamericano de Determinantes Sociales de la Salud. Extraído el 4 de mayo 2014, desde: http://www.alames.org/documentos/ponencias.pdf

Barría, M. (2008). Ministerio de Salud Chile. Determinantes Sociales. Políticas públicas en salud. Extraído el 28 de abril 2014, desde: 
Baum, F. (2007). ¡Salud para todos, ahora! Reviviendo el espíritu de Alma Ata en el siglo veintiuno. Rev Med Soc, 2(1). Extraído el 4 de mayo 2014, desde: http://www.bvsde.paho.org/bvsacd/ cd63/saludtodos.pdf

Behm, H. (2011). Determinantes económicos y sociales de la mortalidad en América Latina. Salud Colectiva, 7(2), 231-253. Extraído el 4 de mayo 2014, desde: http://www.scielo.org.ar/pdf/sc/ v7n2/v7n2a14.pdf

Berlinguer, G. (2007). Determinantes sociales de la enfermedad. Rev Cub Salud Pública, 33(1), 1-7. Extraído el 4 de mayo 2014, desde: http://www.scielosp.org/scielo.php?pid=S086434662007000100003\&script=sci_arttext

Borrell, C. \& Artazcoz, L. (2008). Las políticas para disminuir las desigualdades en salud. Gac Sanit 22(5), 465-73. Extraído el 4 de mayo 2014, desde: http://scielo.isciii.es/pdf/gs/v22n5/politicas. pdf

Declaraciones de World Federation of Occupational Therapists (2011). Extraído el 24 de abril de 2014, desde: http://www.wfot. org/

FLACSO, OPS, MINSAL, OMS, CDSS (2005). Construyendo la nueva agenda social desde la mirada de los determinantes sociales de la salud. Documento síntesis del ciclo de diagnósticos democráticos. Extraído el 4 mayo de 2014, desde: http://cronopio.flacso.cl/fondo/pub/publicos/2005/libro/026065.pdf

Frenz, P. (2005). Desafíos en Salud pública de la Reforma: Equidad y Determinantes Sociales de la Salud. Rev Chil Salud Pública, 9(2), 103-110. Extraído el 4 mayo de 2014, desde: http://www. revistasaludpublica.uchile.cl/index.php/RCSP/article/viewFi$\underline{\text { le/20128/21293 }}$

Guajardo, A. \& Simó S. (2010). Una terapia ocupacional basada en los derechos humanos. TOG (A Curuña) [Internet] 2010 [consultado el 02 mayo 2014]; 7(12):1-25. Disponible en: http:// www.revistatog.com/num12/pdfs/maestros.pdf

Kronenberg, F. \& Pollard, N. (2005). Superar el apartheid ocupacional: exploración preliminar de la naturaleza política de la terapia ocupacional. En: Kronenberg, F.; Simó S. \& Pollard N. Terapia Ocupacional sin Fronteras. Aprendiendo del espíritu de supervivientes. (pp.) Buenos Aires, Editorial Médica Panamericana.

López, O.; Escudero, J.; Carmona, L. (2008). Los determinantes sociales de la salud. Una perspectiva desde el Taller Latinoamericano de Determinantes Sociales de la Salud, ALAMES. Rev Med Soc, 3(4), 323-335. Extraído el 4 de mayo 2014, desde: http://www.socialmedicine.info/index.php/medicinasocial/article/viewFile/260/538

Lysack, C. (2009). Factores Socioeconómicos y su influencia en el desempeño ocupacional. En: Crepeau, E.; Cohn, E. \& Schell, B. (Ed.), Terapia Ocupacional (pp. 68-79). Buenos Aires, Editorial Médica Panamericana.
Morrison, R.; Olivares, D. \& Vidal, D. (2011). La filosofía de la ocupación humana y el paradigma social de la ocupación. Algunas reflexiones y propuestas sobre epistemologías actuales en terapia ocupacional y ciencias de la ocupación. Revista Chilena de Terapia Ocupacional, 11(2), 102-119. Disponible en: http:// www.revistas.uchile.cl/index.php/RTO

Organización Mundial de la Salud (1998). Salud para todos en el Siglo XXI. Extraído el 4 de mayo 2014, desde: http://apps.who. int/gb/archive/pdf_files/EB101/pdfspa/spa8.pdf

Organización Mundial de la Salud (2008). Comisión sobre Determinantes Sociales de la Salud. Resumen analítico del Informe final. Subsanar las desigualdades en una generación. Alcanzar la equidad sanitaria actuando sobre los determinantes sociales de la salud. Extraído el 4 de mayo 2014, desde: http://whqlibdoc. who.int/hq/2008/WHO_IER_CSDH_08.1_spa.pdf

Organizaciones de la Sociedad Civil (2007). Informe de la Sociedad Civil a la Comisión sobre los Determinantes Sociales de la Salud, Organización Mundial de la Salud (CDSS-OMS). Rev Med Soc, 2(4), 212-233. Extraído el 4 de mayo 2014, desde: http:// www.medicinasocial.info/index.php/medicinasocial/issue/ view/14/showToc

Parada, M. (2005). Financiamiento solidario: un espejismo en la reforma sanitaria chilena. Cuad Med Soc (Chile), 45(2):138-144. Extraído el 4 de mayo 2014, desde: http://www.colegiomedico. cl/Portals/0/files/biblioteca/publicaciones/cuadernos/45_2.pdf

Solar, O. \& Irwin, A. (2010). A conceptual framework for action on the social determinants of health. Social Determinants of Health Discussion Paper 2 (Policy and Practice). World Health Organization. Extraído el 4 de mayo 2014, desde: http://www. who.int/sdhconference/resources/ConceptualframeworkforactiononSDH_eng.pdf

Vega, J. (2008). Ministerio de Salud Chile. Subsecretaría de Salud Pública. Próximos desafíos y pasos en Chile. Extraído el 28 de abril 2014, desde:_web.minsal.cl/portal/url/item/ a1d52f772b28681be04001011f0130bc.ppt

Villar, E. (2007). Los Determinantes Sociales de Salud y la lucha por la equidad en salud: desafíos para el Estado y la sociedad civil. Saúde Soc São Paulo, 16(3), 7-13 Extraído el 4 de mayo 2014, desde: http://www.scielo.br/pdf/sausoc/v16n3/02.pdf

web.minsal.cl/portal/url/item/a1d52f772b24681be04001011f0130bc. ppt" 